\title{
Are the Neutrophil-Lymphocyte Ratio and Platelet-Lymphocyte Ratio Prognostic Factors for Patients with Locally Advanced Pancreatic Cancer Treated with Chemoradiotherapy?
}

\author{
Jiong-Jie $\mathrm{Yu}^{1,2}$, Li-Yang Sun ${ }^{1,2}$, Bing Quan ${ }^{1,2}$, and Tian Yang ${ }^{1}$ \\ ${ }^{1}$ Department of Hepatic Surgery, Eastern Hepatobiliary Surgery Hospital, Second Military Medical University, and ${ }^{2}$ Department of Clinical \\ Medicine, Second Military Medical University, Shanghai, China
}

\section{To the Editor:}

We read with great interest the article by Dr. Lee et al. ${ }^{1}$ This retrospective study enrolling 497 patients determined that an elevated pretreatment neutrophil-lymphocyte ratio (NLR) and platelet-lymphocyte ratio (PLR) independently and in combination significantly predicted poor overall survival and progression-free survival in patients with locally advanced pancreatic cancer treated with chemoradiotherapy. Herein, we would like to raise the following comments.

In this study, between patients with normal NLR and elevated NLR and between patients with normal PLR and elevated PLR, significant differences existed in some aspects of patient and tumor characteristics, including subsite of tumor, clinical $\mathrm{T}$ stage, tumor size, resectability and level of CA 19-9 (all p<0.05), suggesting an unbalanced enrollment in the two comparisons. To our knowledge, propensity score matching (PSM) analysis has generally been used in retrospective observational studies, and PSM enables better balancing between groups across all potential risk factors and evaluates the extent of balanced matching using a measurable approach. ${ }^{2,3}$ In 2017, Yang et al. ${ }^{4}$ investigated a similar topic of the association between NLR and prognosis of patients with hepatocellular carcinoma (HCC) after liver resection using PSM analysis and concluded that NLR was an independent prognostic factor for poorer survival in HCC patients undergoing liver resection. Therefore, in view of the higher statistical credibility of PSM analysis, we highly suggest its use.

Furthermore, although the present study contained a certain number of variables on patient and tumor characteristics, it still seemed inadequate. For example, prognostic nutritional index, ${ }^{5}$ plasma fibrinogen levels, ${ }^{5}$ presence of ascites, ${ }^{6}$ serum albu- min levels, ${ }^{7}$ and so forth, were not provided, and these factors might actually influence the prognosis in patients with locally advanced pancreatic cancer treated with chemoradiotherapy and have also been widely covered and studied in previous research. $^{5-7}$

In summary, clarification regarding the omissions mentioned above would greatly solidify the conclusions of the study by Dr. Lee et al. ${ }^{1}$

\section{CONFLICTS OF INTEREST}

No potential conflict of interest relevant to this article was reported.

\section{REFERENCES}

1. Lee BM, Chung SY, Chang JS, Lee KJ, Seong J. The Neutrophillymphocyte ratio and platelet-lymphocyte ratio are prognostic factors in patients with locally advanced pancreatic cancer treated with chemoradiotherapy. Gut Liver 2018;12:342-352.

2. Adamina M, Guller U, Weber WP, Oertli D. Propensity scores and the surgeon. Br J Surg 2006;93:389-394.

3. Hemmila MR, Birkmeyer NJ, Arbabi S, Osborne NH, Wahl WL, Dimick JB. Introduction to propensity scores: A case study on the comparative effectiveness of laparoscopic vs open appendectomy. Arch Surg 2010;145:939-945.

4. Yang T, Zhu J, Zhao L, et al. Lymphocyte to monocyte ratio and neutrophil to lymphocyte ratio are superior inflammation-based predictors of recurrence in patients with hepatocellular carcinoma after hepatic resection. J Surg Oncol 2017;115:718-728.

5. Kurahara H, Maemura K, Mataki Y, et al. Prognostication by

Correspondence to: Tian Yang

Department of Hepatic Surgery, Eastern Hepatobiliary Surgery Hospital, Second Military Medical University, 225 Changhai Road, Shanghai 200438, China

Tel: +86-189-1701-5805, Fax: +86-0-21-81875182, E-mail: yangtianehbh@smmu.edu.cn

Received on May 14, 2018. Accepted on May 21, 2018.

pISSN 1976-2283 eISSN 2005-1212 https://doi.org/10.5009/gnl18210

Jiong-Jie Yu, Li-Yang Sun, and Bing Quan contributed equally to this work as first authors.

@ This is an Open Access article distributed under the terms of the Creative Commons Attribution Non-Commercial License (http://creativecommons.org/licenses/by-nc/4.0) which permits unrestricted non-commercial use, distribution, and reproduction in any medium, provided the original work is properly cited. 
inflammation-based score in patients with locally advanced pancreatic cancer treated with chemoradiotherapy. Pancreatology 2015;15:688-693.

6. Shinoto M, Nakamura K, Shioyama Y, et al. Prognostic significance of a minute amount of ascites during chemoradiotherapy for locally advanced pancreatic cancer. Anticancer Res 2016;36:18791884.

7. Kim HS, Yi SY, Jun HJ, et al. Definitive chemoradiation therapy with capecitabine in locally advanced pancreatic cancer. Anticancer Drugs 2010;21:107-112. 